\title{
Topics
}

\section{Development of Pulse Detection IC for Space LIDAR}

\author{
By Kousuke KawaHARA, Hirokazu IKedA and Takahide Mizuno \\ The Institute of Space and Astronautical Science, JAXA, Sagamihara, Japan
}

(Received July 17th, 2009)

\begin{abstract}
In recent years, LIDAR has been used in remote sensing systems, obstacle avoidance systems on planetary landers, rendezvous docking systems, and formation flight control systems. A wide dynamic range is necessary for LIDAR systems on planetary landers and in rendezvous docking systems. For example, a dynamic range of $60 \mathrm{~dB}(60 \mathrm{~dB}$ for light, $120 \mathrm{~dB}$ for electric signal) was required for the receiving system used in the Hayabusa mission in order to measure distances between $50 \mathrm{~m}$ and $50 \mathrm{~km}$. In addition, rendezvous docking systems require a ranging resolution of better than $10 \mathrm{~cm}$. For planetary landers and rendezvous docking systems, ISAS/JAXA is developing a customized integrated circuit (IC) for LIDAR reception. This report introduces the design of the customized IC and reports the results of preliminary experiments evaluating the prototype, LIDARX02.
\end{abstract}

Key Words: Laser, Altimeter, LIDAR, ASIC, Planetary Explorer

\section{Introduction}

In recent years, planetary exploration missions aiming to elucidate the origin of the solar system have been conducted, such as Hayabusa (Japan), NEAR (USA), and Rosetta (Europe). Many of the explorers currently in operation or planned for observation of the moon and planets, such as SELENE (SELenological and ENgineering Explorer), the Lunar Reconnaissance Orbiter, Messenger, Bepi-Colombo, and the Mars Global Surveyor, incorporate LIDAR (LIght Detection And Ranging) as a critical navigation sensor for long-range measurements ${ }^{1-7)}$. The LIDAR system enables planetary explorers to measure distance from the target planet.

The receiving circuit of Hayabusa's LIDAR consists of discrete electric devices. In the present study, we aim to reduce the circuit area and shorten the development period, by using techniques for fabricating sub-micron analog integrated circuits (ICs) that were developed in the field of high-energy physics. Some time-to-digital converters (TDC) that interpolate a digital clock counter for a detected timing are already commercially available ${ }^{8)}$. However, the proposed device also incorporates a circuit for timing detection and an interpolator. In addition, the proposed device must cover the wide signal dynamic range that is particular to planetary explorers. In this paper, we first report problems that have been revealed through the actual operation of Hayabusa's LIDAR. Then, we discuss the required performance and functionality for LIDAR receiving circuits of planetary explorers. After this introduction, we present the design of a customized IC and report the current state of its development.

\section{Experience of Hayabusa Mission}

First, we focus on the receiving circuit of Hayabusa's LIDAR as a representative example of LIDAR for a planetary explorer. A laser pulse scattered on an asteroid's surface is detected with an avalanche photodiode (APD) of the LIDAR system. This detected signal is amplified by a preamplifier and converted to a bipolar wave by a pulse-shaping amplifier. A comparator detects the zero-cross timing of the bipolar wave to measure signal timing. The transmitted pulse and the incoming pulse are detected using the same pulse-detecting circuit in order to reduce thermal fluctuations. A digital counter for ranging is started by the transmitted pulse and stopped by the incoming pulse. By using the leading edge and trailing edge of the digital clock of $75 \mathrm{MHz}$, a ranging resolution of $\pm 1 \mathrm{~m}$ is obtained.

The ranging coverage of the system depends on the reflection of the asteroid's surface. Assuming that asteroids have a perfect diffusion surface and their reflections are only $5 \%$ (hemispherical reflectance: $10 \%$ ), the estimated coverage of ranging is $50 \mathrm{~m}$ to $50 \mathrm{~km}$. The distance from $50 \mathrm{~m}$ to $50 \mathrm{~km}$ corresponds to a dynamic range of $60 \mathrm{~dB}$ for the power of the incoming signal. A wide dynamic range is one of the critical functions for the LIDAR system that is loaded on the asteroid explorer Hayabusa.

In order to cover this wide dynamic range, Hayabusa's LIDAR has 8 levels in its gain control system. It can change gain by adjusting the bias voltage of the APD in 3 steps and changing the preamplifier gain in 3 steps. Through these combinations, a dynamic range of $40 \mathrm{~dB}$ for LIDAR has been achieved. Since the approach velocity of Hayabusa is very slow, for example, several centimeters per second to several tens of centimeters per second, the time constant of the auto gain control (AGC) is $100 \mathrm{~s}$.

Hayabusa's LIDAR can adjust gain in 6-dB steps by adjusting the bias voltage of the APD and the feedback capacitances of the charge amplifier in this design. Due to inadequate adjustment before flight, one of the gain stages has a gain gap of over $10 \mathrm{~dB}$. This caused hunting when the gain stage was switched. The effect of this phenomenon was rather slight, and thus it did not impact the touchdown operation of Hayabusa. In addition, insufficient calibration made the signal level ambiguous when the gain stage was changed. Neither of these problems affected the touchdown operation, although improvement is necessary for future missions.

\section{Calculations}

If the distance to a target is $\mathrm{R}$, the received signal power is given by 


$$
P_{r}=\frac{P_{t} G_{t}}{4 \pi R^{2}} \sigma \frac{1}{4 \pi R^{2}} \pi\left(\frac{D}{2}\right)^{2} \eta
$$

The symbols are defined as follows.

$\mathrm{P}_{\mathrm{r}}$ : incoming signal power

$\mathrm{P}_{\mathrm{t}}$ : transmitted signal power

$\mathrm{G}_{\mathrm{t}}$ : power gain of transmitting antenna

D: diameter of receiving antenna

$\mathrm{R}$ : distance to target

$\sigma:$ target cross-sectional area

$\eta$ : system efficiency

If the beam width of the transmitting laser and the reflectance are defined as $\theta$ and $\rho$ (for isotropic target), respectively, $\sigma$ is given by

$$
\sigma=2 \rho \pi\left(\frac{R \theta}{2}\right)^{2}
$$

The relational between $\mathrm{G}_{\mathrm{t}}$ and $\theta$ is given by

$$
G_{t}=\frac{4 \pi}{\theta^{2}} .
$$

Then, the incoming power can be written as follows.

$$
P_{r}=P_{t} \frac{\pi \rho D^{2}}{32 R^{2}} \eta \text {. }
$$

We assume that the transmitting energy $\left(\mathrm{P}_{t}\right)$ is $5 \mathrm{~mJ}$, the wavelength of the laser is $1.064 \mu \mathrm{m}$, the transmitted pulse width is $10 \mathrm{~ns}$, the target reflectivity $(\rho)$ is $5 \%$, the diameter of the receiving antenna is $100 \mathrm{~mm}$, the system efficiency is $70 \%$, and the distance from the target is $50 \mathrm{~m}$. In this case, the incoming signal energy is $6.87 \times 10^{-11} \mathrm{~J}$, as calculated with Eq. (4). The corresponding number of photons is $1.5 \times 10^{10}$. Under the conditions described above, the efficiency and the multiplication of the APD are $40 \%$ and 100 , respectively. In the case of $\mathrm{R}=50 \mathrm{~km}$, the incoming signal energy becomes $6.87 \times 10^{-17} \mathrm{~J}\left(1.5 \times 10^{4}\right.$ photons $)$. When the target distance changes from $50 \mathrm{~m}$ to $50 \mathrm{~km}$, the incoming electrical charge changes from $2400 \mathrm{pC}$ to $0.0024 \mathrm{pC}$.

\section{Required Specifications}

The receiving circuit of LIDAR used for a non-cooperative target (e.g., landing and rendezvous docking) is required to have a large dynamic range as mentioned above. We are developing a customized IC that has a dynamic range of $60 \mathrm{~dB}$ for planetary explorers. During actual operation, the back scattering factor will change depending on the incident angle of the laser and fluctuation of the irradiated spots. Thus, the incoming power of each shot will vary widely. Therefore, each gain stage will need to have a dynamic range of about $10 \mathrm{~dB}$. In addition to this power variance of the incoming signal, the pulse width of the reflection is also expected to fluctuate widely. Therefore, the transmitted pulse width needs to be a few to ten nanoseconds, and the receiving circuit needs to accept pulses that have pulse widths from ten to several hundreds of nanoseconds.

Since Hayabusa's LIDAR does not have a clock interpolator, the resolution of the ranging is decided by the clock of the $75-\mathrm{MHz}$ digital counter. For this reason, its resolution was $\pm 1 \mathrm{~m}$. Scientific observations and navigation systems require improved resolution; however, improvements in the time resolution by adopting a higher frequency digital clock are not possible considering chip fabrication, screening tests, and the power consumption of the digital device. Therefore, a new device that employs a digital clock below about $20 \mathrm{MHz}$ and adopts a clock interpolator is necessary in order to obtain higher resolutions.

\section{Design of Proposed Device}

The main purpose of this development is to manufacture a customized IC by using submicron analog IC technology that has been developed in the field of high-energy physics ${ }^{9}$. The procedure for fabrication is the Taiwan Semiconductor Manufacturing Company (TSMC) complementary metal-oxide semiconductor (CMOS) $0.35-\mu \mathrm{m}$ process that allows dependable manufacturing, with which we have considerable experience. The size of the bare chip is $3 \times 3$ $\mathrm{mm}^{2}$. The package is a ceramic quad flat package (QFP) with a size of $14 \times 14 \mathrm{~mm}^{2}$ ( 80 pins). The development process is described below.

1. Design of circuit

2. Evaluation of circuit with SPICE simulations

3. Layout design and extraction of parasitic capacitance

4. Evaluation of circuit with SPICE simulations

5. Device manufacturing and packaging

6. Evaluation tests

Except for processes 3 and 5, the other processes use in-house technology. A photograph of the prototype bare IC chip referred to as LIDARX02 is shown in Figure 1.

Figure 2 shows the circuit structure of LIDARX02. LIDARX02 consists of a divider module, an integrator module, a timing detector module and a time-to-analog converter (TAC). The APD is located on an external test board and converts light signals to electrical charge. As mentioned above, there is a difference of about $60 \mathrm{~dB}$ between the signal levels of short-range and long-rang measurements.

The divider consists of a T-shaped circuit with a capacitor that is mounted on the outside of the device and divides a large quantity of electrical charge at short range into a suitable amount of charge. The dividing ratio of charge can be decided based on the ratio of internal and external capacitors. The combinations of external and internal capacitors are selectable with mechanical switches. Consequently, the device has five input channels through the various combinations of the dividers and the integrator. Therefore, the device has five steps of coarse adjustment for the gain. Each channel (CH0-CH4) has a gain range with a factor of 16 , and the total dynamic range of the device reaches $60 \mathrm{~dB}\left(16^{5} \fallingdotseq 10^{6}\right)$.

The integrator consists of a filter and amplifiers that can change the gain by changing the value of the feedback capacity. The feedback capacity can be changed with a 4-bit command. Using this function, the gain of the integrator can undergo fine adjustment. The compensation circuit of the pole/zero is installed on the stage next to the integrator. This improves the symmetry of output waves (leading) by adjusting a time constant of the amplifier and a time constant of the 
decay. As a result, the symmetry of the differential waveform that is generated in a subsequent stage after the bipolar conversion is also improved. The symmetry of the differential wave reduces the dependence of the detection timing on the input signal level.

The timing detector transforms the leading wave to a bipolar wave, which is referred to as a differential wave, and detects its zero-cross timing as the pulse detection timing. In order to prevent false detections caused by noise overlap on the differential wave, a threshold is set up for the leading wave; thus, only when the pulse height of the leading wave exceeds the threshold level that is set by external voltage, the HIT signal is generated at the zero-cross timing. The main functions of this device are its $60-\mathrm{dB}$ dynamic range with the divider and the integrator and timing detection using the timing detector.

The TAC is activated by the HIT signal generated in the timing detector. Figure 3 shows the principles of the timing measurement. From the TAC, a saw wave (about $4 \mathrm{mV} / \mathrm{ns}$ ) such as the one shown in Figure 3 begins to rise upon detection of the leading edge of the HIT signal. Then, the analog voltage of the saw wave is held at the timing of STOP signals synchronized with the digital clock and output the held voltage. The zero-cross point (Figure 3) is estimated from the analog voltage of the TAC.

In order to estimate the zero-cross timing, the inclination of the saw wave is highly important. However, the inclination has the possibility of changing due to temperature and the deterioration with age. Therefore, the TAC produces an analog voltage twice. As a result of this function, the change in the inclination of the saw wave does not influence the estimation.

In the evaluation system (Figure 4 and Figure 5), the clock of the digital controller is $15.625 \mathrm{MHz}$, and thus the interval time of STOP signals is $64 \mathrm{~ns}$. Since the timing estimation uses analog voltage, the resolution of interpolation depends on the resolution and the accuracy of analog-to-digital converter (ADC) mounted on an external test board.

This device has a test port (TP) in order to evaluate the internal circuit without an APD. The TP is directly connected to the integrator by an internally mounted capacitor. Thus, a voltage step can be substituted for the signal from the APD.

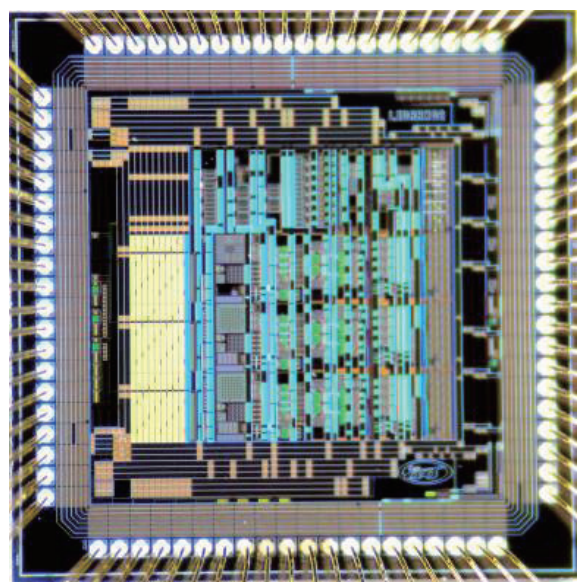

Fig. 1. Bare chip of prototype IC (LIDARX02).

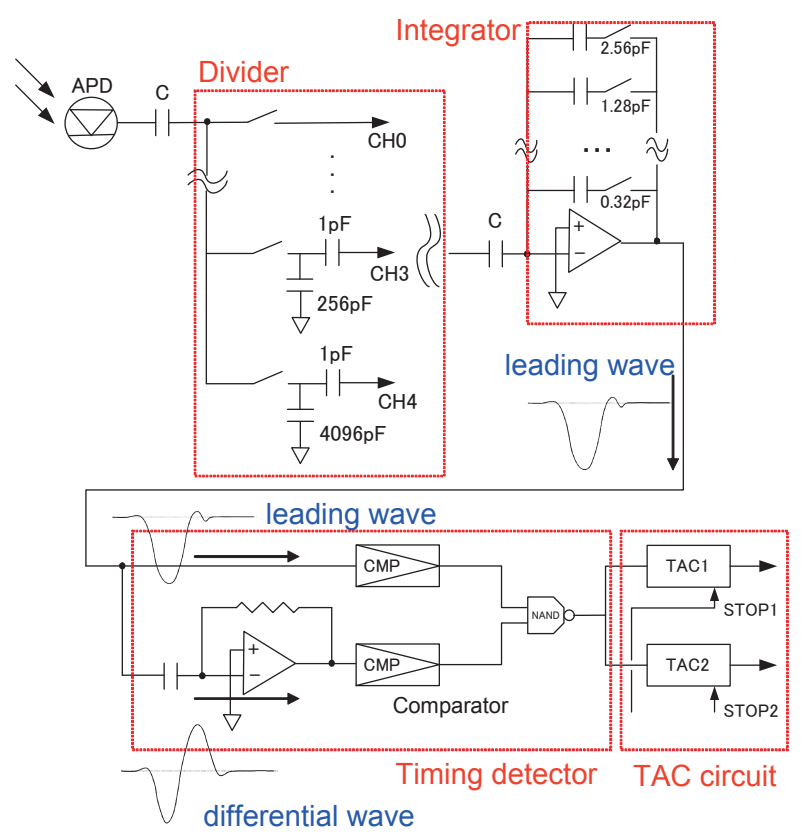

Fig. 2. Circuit structure (LIDARX02).

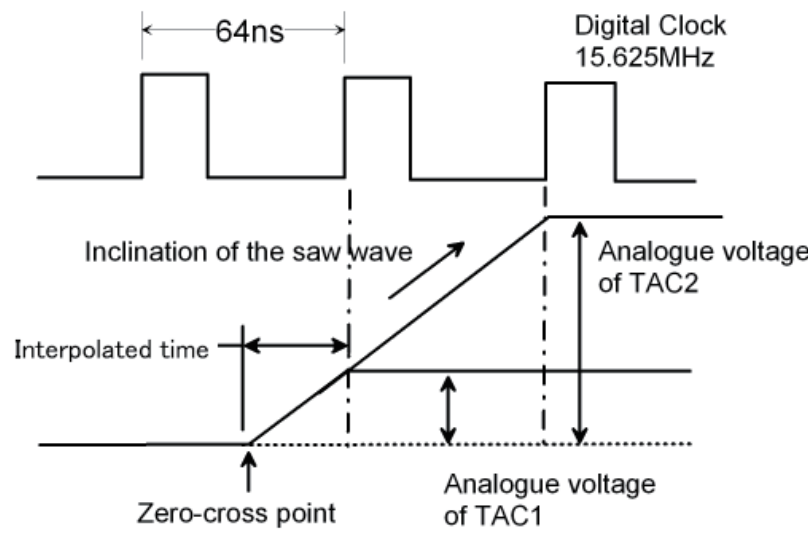

Fig. 3. Timing measurement principle.

\section{Evaluation System}

A comprehensive evaluation test of the device (LIDARX02) should be done by using a light pulse on an external APD. However, in this paper as a preliminary test, we evaluated this device by using an electrical charge pulse from an AC-coupled test port (TP) instead of an APD. Figure 4 and Figure 5 show a block diagram of the evaluation system and its photograph. The evaluation system mainly consists of a digital controller, the test board for the proposed device and a PC.

The digital controller has functions to generate various timings such as emission of a laser, detection of a HIT signal and TAC circuit control. Furthermore, it controls the counter for time of flight (TOF), commands for the device and telemetry for the PC. The test device is mounted on the LIDARX02 test board. Various thresholds for the signal detection are inputted to the board. The PC can set various parameters of the test device from the console display that is shown in Figure 6 . The console display shows telemetry data 
such as digital counter output and the output voltage of the TAC. HIT timing estimation is calculated by the PC.

The digital controller sends all digital signals for device setup to the device via an LVTTL (Low Voltage Transistor-Transistor Logic) interface. The interface for timing signals such as the HIT signal and TAC control signals are LVDS (Low Voltage Differential Signaling) (400 mV \pm $200 \mathrm{mV}$ ). The interface between the digital controller and the $\mathrm{PC}$ is RS232C.

The pulse generator sends a delayed pulse triggered by the digital controller that simulates a reflected pulse from a target. A voltage step generated by the pulse generator is input from the TP through a step attenuator. The step attenuator can control electrical charges, so this evaluation system can simulate LIDAR measurements for a range from $50 \mathrm{~m}$ to 50 $\mathrm{km}$.

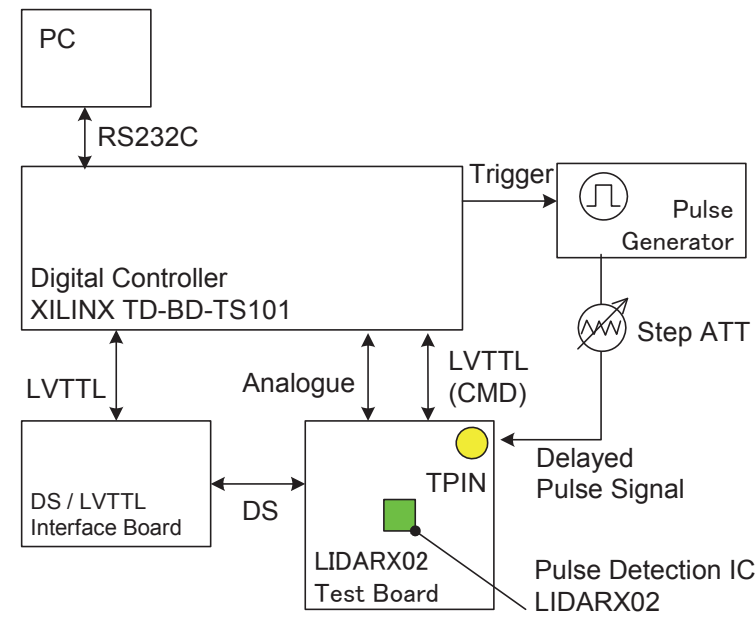

Fig. 4. Block diagram of evaluation test system.

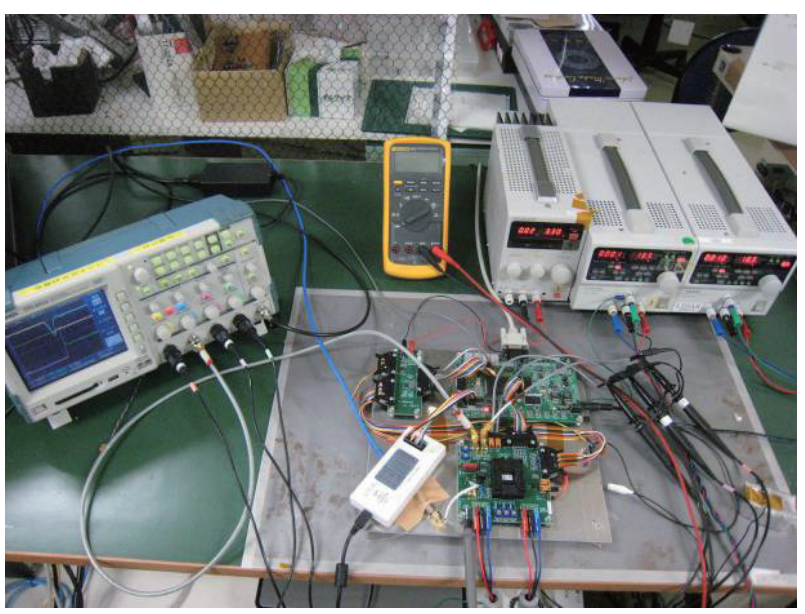

Fig. 5. Evaluation test system.

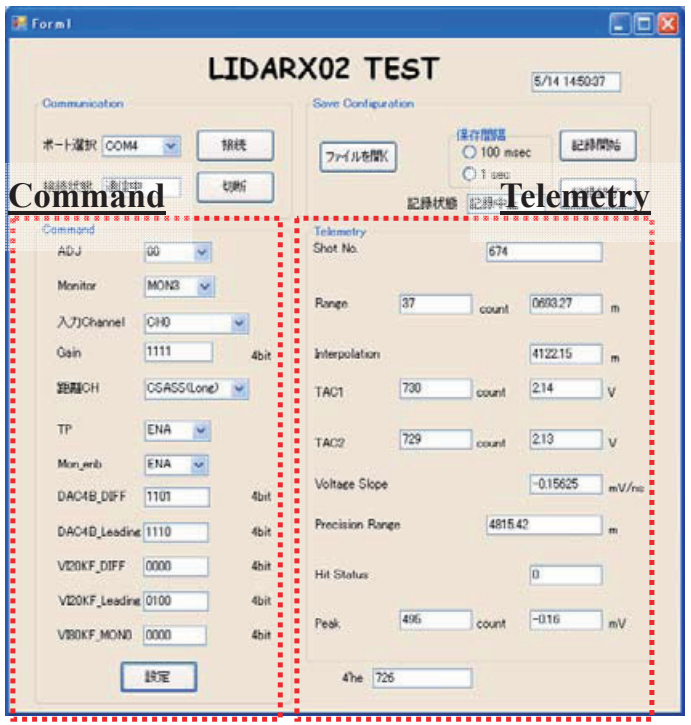

Fig. 6. Console display.

\section{Results of Evaluation}

Figure 7 shows typical output waveforms from LIDARX02. The top waveform is a bipolar wave that is transformed from a leading wave. The timing detector detects the zero-cross timing of this waveform and generates a HIT signal (second waveform). The saw wave from the TAC starts rising upon being triggered by the leading edge of the HIT signal. The amplitude of the saw wave is generated twice according to the timing of the digital clock following the HIT signal. These two analog values are output as TAC1 (third waveform) and TAC2 (forth waveform). The HIT timing is estimated with $\mathrm{TAC} 1$ and TAC2. Therefore, stability and repeatability are important for measuring TOF. The zero-cross timing of bipolar waves must be stable, because this is the origin of the HIT signal.

In the case of actual TOF measurement for non-cooperative targets, the input level of the receiving optics fluctuates. LIDARX02 achieves a wide dynamic range via the coarse adjustment function of the divider (select $\mathrm{CH}$ ) and the fine adjustment function of the integrator (select gain). Therefore, the relatively slow fluctuation of the input signal caused by the distance from a target does not cause problems. However, fluctuation of the level of a reflected signal level caused by local geographical and geological features and the incident angle of the laser is relatively fast. Thus, the device needs a wider dynamic range without gain adjustment. Figure 8 shows the input charge dependence of the HIT timing and the leading wave amplitude in $\mathrm{CHO}$. $\mathrm{CHO}$ is designed the long-distance measurements, and thus has high gain and is comparatively unstable. The gain setting (integrator setting) of $\mathrm{CHO}$ is set to the minimum. Figure 8 shows the relation between the HIT signal timing and the input charge level when the input charge is varied from $0.01 \mathrm{pC}$ to $0.1 \mathrm{pC}$. Within the range from $0.01 \mathrm{pC}$ to $0.05 \mathrm{pC}$, the leading amplitude increases from $150 \mathrm{mV}$ to $400 \mathrm{mV}$. In this range, the repeatability of timing detection is stable within several ns. Also, the result corresponds to the SPICE (Simulation Program with Integrated Circuit Emphasis) simulation. In this 
measurement, the timing of the HIT signal was measured by an oscilloscope. Since the cursor resolution is only 2 ns, this result contains a comparable error level. Since the result has high repeatability, the measurement can reach sub-nanosecond-level accuracy if we construct a suitable calibration curve. After constructing a calibration curve, it will be necessary to further improve the resolution of the measurement system and further evaluate the system. In addition, we consider it likely that we can achieve an accuracy level of several nanoseconds in a range of greater than $0.05 \mathrm{pC}$ by constructing a calibration curve.

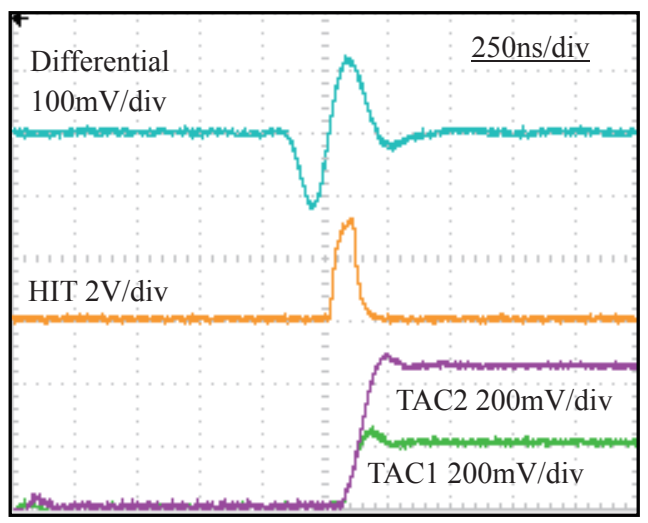

Fig. 7. Typical output waveforms from LIDARX02.

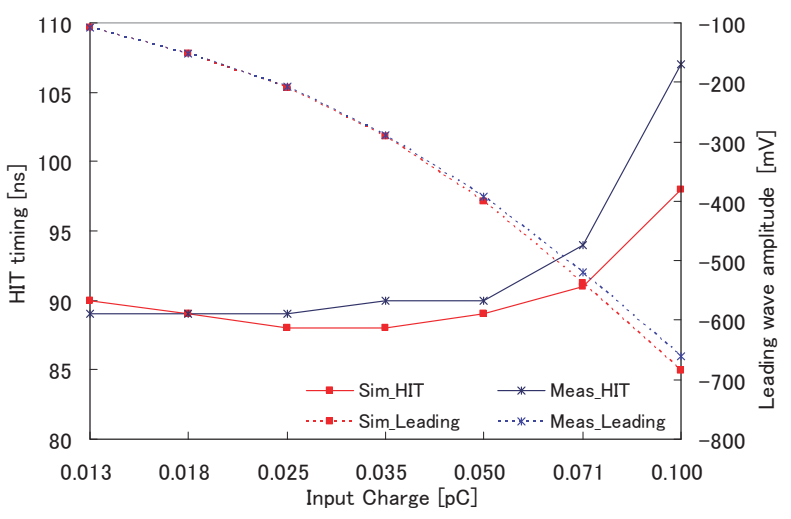

Fig. 8. Stability of zero-cross timing.

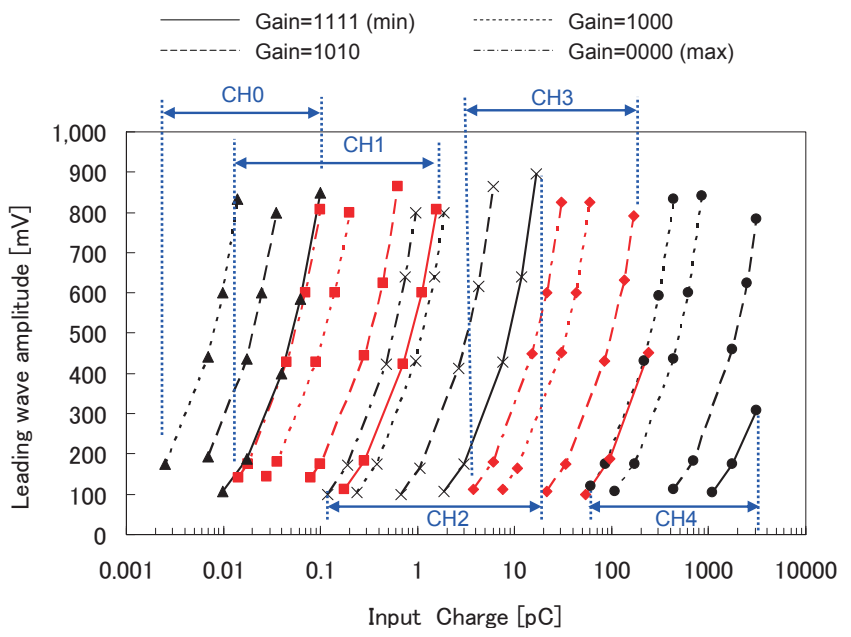

Fig. 9. Relation of input charge and leading wave amplitude.
The main function of the proposed device is timing detection of light pulses in a $60-\mathrm{dB}$ dynamic range at the input level. In order to evaluate this function, we measured the behavior of this device by changing the input electrical charge. The amount of input charge changed from $0.002 \mathrm{pC}$ to $3000 \mathrm{pC}$. Figure 9 shows the relation between input charge and the amplitude of the leading wave. The gain setting of the integrator was at a typical setting for each channel.

Figure 9 shows that the dynamic range of this device is about $60 \mathrm{~dB}$; in fact, the minimum input charge level is 0.002 $\mathrm{pC}$ and the maximum level is $3000 \mathrm{pC}$. Furthermore, each channel has a dynamic range of over $10 \mathrm{~dB}$ and the channels overlap. Therefore, the device can conduct measurements over the entire 60-dB dynamic range. In Figure 9, the device feature is illustrated by the input charge that corresponds to the input light power. However, if it is illustrated on the horizontal axis of the electric signal power, the device has the dynamic range of $120 \mathrm{~dB}$. The amplitude of the leading wave is between $100 \mathrm{mV}$ to $900 \mathrm{mV}$. In this range, the HIT signal is output normally. However, according to the result shown in Figure 8 , the range in which the HIT signal is comparatively independent from the amplitude of the leading wave is from $150 \mathrm{mV}$ to $400 \mathrm{mV}$. Therefore, in order to obtain a good quality timing signal, the gain settings must be adjusted to limit the amplitude of the leading wave within this range. As shown in Figure 9, if the amplitude of the leading wave is limited to the range of $150 \mathrm{mV}$ to $400 \mathrm{mV}$, the coverage of each channel is overlapping and the device still obtains a 60-dB dynamic range.

\section{Conclusion}

The background, design and evaluation of a prototype IC for LIDAR pulse detection are reported in this paper. According to the evaluation results, all the main functions of the IC for pulse detection in a $60-\mathrm{dB}(60 \mathrm{~dB}$ for light, $120 \mathrm{~dB}$ for electric signal) dynamic range, the pulse timing generation, and the interpolation of the digital clock were confirmed to operate effectively. This device can cover from $0.002 \mathrm{pC}$ to $3000 \mathrm{pC}$ by five Divider's channels and four Integrator's gains. It also achieved the timing accuracy of nano-seconds. One setting of Integrator's gain has a dynamic range of $8 \mathrm{~dB}$ that is enough wide for an auto gain control of receiver. In the future, we plan to improve the resolution of the measurement system and comprehensively evaluate this device using light pulses.

\section{References}

1) Mizuno, T., Tsuno, K., Okumura, E. and Nakayama, M.: LIDAR for Asteroid Explorer Hayabusa: Development and Onboard Evaluation, JJSASS, 54 No. 634 (2006), pp. 514-521.

2) Cole, T. D., Boies, M. T. and El-Dinary, A. S.: Laser Radar Instrument for the Near-Earth Asteroid Rendezvous (NEAR) Mission, Proceedings of SPIE - The International Society for Optical Engineering, 2748 (1996), pp. 122-139.

3) Araki, H., Tazawa, S., Noda, H., Tsubokawa, T., Kawano, N. and Sasaki, S.: Observation of the Lunar Topography 
by the Laser Altimeter LALT on Board Japanese Lunar Explorer SELENE, Advances in Space Research, 42 no. 2 (2008), pp 317-22.

4) Riris, H., Sun, X., Cavanaugh, J. F., Jackson, G. B., Ramos-Izquierdo, L., Smith, D. E. and Zuber, M.: The Lunar Orbiter Laser Altimeter (LOLA) on NASA's Lunar Reconnaissance Orbiter (LRO) Mission, Proceedings of SPIE - The International Society for Optical Engineering, $\mathbf{6 5 5 5}$ Sensors and Systems for Space Applications (2007), p. $65550 \mathrm{I}$.

5) Cavanaugh, J. F., Smith, J. C., Sun, X., Bartels, A. E., Ramos-Izquierdo, L., Krebs, D. J., McGarry, J. F., Trunzo, R., Novo-Gradac, A. M., Britt, J. L., Karsh, J., Katz, R. B., Lukemire, A. T., Szymkiewicz, R., Berry, D. L., Swinski, J. P., Neumann, G. A., Zuber, M. T. and Smith, D. E.: The Mercury Laser Altimeter Instrument for the MESSENGER Mission, Source: Space Science Reviews, The MESSENGER Mission to Mercury 131 no. 1-4, (2007), pp. 451-479.

6) Murphy, E. M., Rando, N., Falkner, P. and Peacock, A.: LAPE: Laser Altimeter for Planetary Exploration, Proceedings of the SPIE - The International Society for Optical Engineering, 5240 no. 1 (2004), pp. 10-16.

7) Ramos-Izquierdo, L., Bufton, J. L. and Hayes, P.: Optical System Design and Integration of the Mars Observer Laser Altimeter, Applied Optics, 33 No. 15 (1994), pp. 307-322.

8) ATLAS-JAPAN: http://atlas.kek.jp/tdc

9) Manobu, T., Hirokazu, I., Mitsuo, I. and Susumu, I.: Performance of a Monolithic Time-to-Amplitude Converter for High Precision TOF Measurements, Nuclear Instruments and Methods in Physics Research A312 (1992), pp. 585-590. 